\section{Clima laboral, Burnout y Perfil de Personalidad: Un estudio en personal asistencial de un Hospital Público de Lima ${ }^{6}$}

Miguel Ángel Vizcarra Fernández
Médico, Especialista en Neurocirugía
EsSalud
Lima, Perú
Correo: miguel.vizcarra@essalud.gob.pe

Victoria Llaja Rojas

Doctora en psicología Universidad Nacional Mayor de San Marco

Lima, Perú

Correo: llaja1@gmail.com

Consuelo Limo Sánchez

Mg. en Epidemiología Jefe de Enfermería EsSalud Lima, Perú.

Correo: consuelolimo@hotmail.com

Johan Talavera Aguilar

Licenciado en Psicología Clínica Universidad Nacional Mayor de San Marco

Lima, Perú

Correo: jta159102@gmail.com

\title{
Resumen
}

Objetivo: relacionar el clima laboral con el Síndrome de Burnout, y el perfil de Personalidad frecuente en las áreas de trabajo neuroquirúrgicas donde los estresores son de alta incidencia por el tipo de paciente, la necesidad de intervenir en situaciones de crisis y el exceso de trabajo para realizar las actividades programadas. Método: La muestra fue intencional, en un hospital público de Lima, se evaluó a 30 profesionales de salud, administrando la escala estandarizada de Clima Laboral, Scanning Neuropsicológico y el Inventario de Burnout. Resultados: En burnout, un $80 \%$ no presenta dicho síndrome, $10 \%$ con tendencia y $10 \%$ en riesgo. Existiendo un Clima Laboral medio (60.0\%), el área de Condiciones Laborales es desfavorable. El perfil de personalidad un $90 \%$ es equilibrada, $10 \%$ de tipo personalidad con firmeza de decisión. Conclusiones: Existe correlación positiva estadísticamente significativa entre clima laboral y Burnout. Se discuten los hallazgos y se asociaron a sus repercusiones en trabajadores de salud.

Estrés laboral, Scanning Neuropsicológíco, personalidad, autorrealización, involucramiento laboral, supervisión.

6 Para Citar este artículo: Vizcarra, M., Llaja, V., \& Talavera, J. (2015). Clima laboral, Burnout y perfil de personalidad: un estudio en personal asistencial de un hospital público en Lima. Informes Psicológicos, 15(2), 111- 126. http://dx.doi. org/10.18566/infpsicv15n2a06 


\section{Working environment, burnout and personality profile: A study in the nursing staff of a public hospital in Lima}

Abstract

Aim: To relate the work environment with Burnout Syndrome and the personality profile present in neurosurgical workspaces, where stressors are of high incidence due to patient type, the need to intervene in crisis situations and overwork for scheduled activities. Methods: The sample was intentional, in a public hospital in Lima; 30 health professionals were evaluated, by administering the standardized scale of work environment, Neuropsychological Scanning and the Burnout Inventory. Results: In burnout, $80 \%$ do not present such syndrome, $10 \%$ are in the trend and $10 \%$ at risk; there is a medium-level working environment $(60.0 \%)$, the area of working conditions is unfavourable; the personality profile is $90 \%$ balanced, kind personality with firmness of decision found in $10 \%$. Conclusions: There is a statistically significant positive correlation between employee satisfaction and burnout. Findings are discussed and are associated with their impact on health care workers.

\section{Clima laboral, Burnout e Perfil de Personalidade: Um estudo em pessoal assistencial de um Hospital Público de Lima}

Objetivo: Relacionar o clima laboral com a Síndrome de Burnout, e o perfil de Personalidade frequente nas áreas de trabalho neurocirúrgicas onde os estressores são de alta incidência pelo tipo de paciente, a necessidade de intervir em situações de crise e 0 excesso de trabalho para realizar as atividades programadas. Método: A amostra foi intencional, num hospital público de Lima, se avaliou a 30 profissionais de saúde, administrando a escala padronizada de Clima Laboral, Scanning Neuropsicológico e 0 Inventário de Burnout. Resultados: Em burnout, um 80\% não apresenta dita síndrome, 10\% com tendência e 10\% em risco, existe um Clima Laboral meio (60.0\%), a área de Condições Laborais é desfavorável; o perfil de personalidade um $90 \%$ é equilibrada, $10 \%$ de tipo personalidade com firmeza de decisão. Conclusões: Existe correlação positiva estatisticamente significativa entre clima laboral e Burnout. Se discutem as descobertas e se associaram a suas repercussões em trabalhadores da saúde. 


\section{ntroducción}

Los profesionales de instituciones de servicios sociales, sanitarios y educativos se ven forzados a implicarse durante muchas horas en los problemas y preocupaciones de las personas con las que se relacionan profesionalmente. Frecuentemente, la interacción del profesional con el sujeto, se centra en los problemas actuales de éste (salud, higiene mental, relaciones personales o laborales, etc.) y éstos vienen cargados con diversos sentimientos, tales como la preocupación, miedo, rechazo, odio, desesperación, etc. Por otra parte, como no siempre es obvia la solución a estos problemas esa interacción se hace ambigua y frustrante. En consecuencia, el profesional que continuamente trabaja con personas en esa labor asistencial, va acumulando un estrés crónico que puede cansarle emocionalmente y, finalmente, llevarle a una situación de agotado o quemado; vale decir, este síndrome puede aparecer principalmente en profesionales que trabajan con personas y se implican en los problemas de éstas Rodríguez (1995).

Este síndrome conlleva los síntomas siguientes: Agotamiento emocional, despersonalización y disminución de la realización personal y puede aparecer principalmente en profesionales que trabajan con personas y se implican en los problemas de éstas. Las consecuencias de este síndrome, son potencialmente de alto riesgo para este personal, para los usuarios que son atendidos por ellos y para la institución en que trabaja.
La literatura refiere que el clima laboral $u$ organizacional es determinante en la aparición de diferentes formas de estrés ocupacional, y entre ellos el Síndrome de Burnout que es conocido en el ámbito clínico-asistencial, como una forma de estrés crónico que puede afectar a los profesionales de la salud ya que ellos atienden de manera permanente problemas emocionales o preocupaciones diarias de los receptores de su servicio: los pacientes en situación de enfermedad aguda o crónica (Rodríguez, 1995).

En el estudio realizado por Vásquz, Maruy y Verne (2014) donde participaron 54 trabajadores entre médicos (25), enfermeras (16) y técnicos de enfermería (13), se encontró una frecuencia de Burnout de 3,76\%; presentaron un nivel alto de cansancio emocional (12,97\%), un nivel alto de despersonalización (11.12 \%) y el 14,81\% presentó bajo nivel de realización personal. Concluyeron que la falta de trabajo del cónyuge, sexo, condición laboral y tiempo de trabajo tuvo relación estadísticamente significativa con las dimensiones de este síndrome.

Así mismo en el reporte hecho por Sánchez y Sierra (2014) en una muestra de 68 enfermeros y auxiliares que trabajan en la UVI del Hospital de Ramón y Cajal se encontraron bajas puntuaciones en las tres dimensiones del síndrome (cansancio emocional, despersonalización y realización personal), y tendencia a padecerlo la mayoría de la población un 45,6\%. Se concluyó que el perfil del trabajador que padece síndrome de Burnout sería en primer lugar las enfermeras, de 35 a 44 años, con contrato fijo, experiencia laboral mayor de 16 años y una antigüedad de 1 a 5 años en el puesto de trabajo (Gamonal, García, \& Silva, 2008). 
Referente a estudiantes universitarios del séptimo año de Medicina de una universidad privada de Lima se encontró que la prevalencia de Burnout en el 57 $\%$ de los examinados. Más de la mitad de la población presentó niveles medio o alto en despersonalización (DP) 68,9\% y agotamiento emocional $67 \%$ y aproximadamente un tercio de los estudiantes tuvieron baja realización personal (RP) 35\%. No se encontró asociación estadísticamente significativa entre las variables sociodemográficas y laborales estudiadas y la presencia de Síndrome de Burnout (Villa, 2010).

En estudios con profesionales de la salud, específicamente enfermeras (Llaja \& Muñoz, 2000) donde se evaluó el nivel de Estrés tanto en el personal de $\mathrm{UCl}$ del 7 mo. B como de UCIN, con una muestra de 60 enfermeras, cuyos instrumentos utilizados fueron las Escalas de Estrés de Valverde y el Registro de Evaluación Neuropsicológica POC- H.N.E.R.M; se concluyó que el $45 \%$ de los casos se encuentra en un nivel de estrés alto y el 43.3\% en término medio, lo que significaba que más de la mitad del Personal de Enfermeras estaba en alto riesgo de presentar el Síndrome de UCl. Asimismo, se determinó que el nivel de estrés acumulado más alto se encontraba en el personal de Enfermería de UCI 7B.

Capa (2004) desarrolló la investigación de estrés laboral asistencial con 440 profesionales de la salud psicólogos, médicos tecnólogos, enfermeras, obstetras, odontólogos y trabajadores sociales de centros hospitalarios de Lima Metropolitana; se administró el Inventario de Maslach (MBI) y el Cuestionario de Factores Psicosociales. Se empleó el paquete SPSS versión 11 en castellano y el programa multivariante de Ecuaciones Estructurales EQS versión 5.7 para Windows. Uno de los hallazgos importantes fue que en la muestra total estudiada presenta Burnout en un $44 \%$ lo que llevó a afirmar que el nivel de prevalencia del Síndrome de Burnout en los profesionales de la salud estudiados fue alto. De acuerdo con la teoría de Maslach y Jackson (1986), los sujetos con Burnout son quienes presentan niveles altos en cansancio emocional y despersonalización y nivel bajo en realización personal. Igualmente, el 16\% de los profesionales presentaron algún grado de riesgo o tendencia al Burnout. Estos resultados indicaron que los profesionales más afectados por el estrés laboral asistencial son las obstetras y los menos afectados son los Trabajadores Sociales.

En otro estudio, sobre percepción del clima Laboral y de la eficiencia personal en profesionales de la salud del sector público de la ciudad de Lima (Bulnes, 2004) realizado a 93 profesionales de centros hospitalarios públicos de Lima, en el cual se usó la escala de clima social laboral de Moos y Moos (1974) y el $\mathrm{MBI}$ de Maslach \& Jackson, no se evidenció correlación entre las Escalas de Estrés laboral y la Escala de clima social laboral en la muestra estudiada; por otra parte los médicos, enfermeras y tecnólogos médicos son los que presentan una mayor disposición, como consecuencia de su actividad profesional, de presentar un mayor nivel de estrés o agotamiento profesional y en lo que respecta al tiempo de servicio, los profesionales con un tiempo de servicio entre 25 y 32 años, el nivel de estrés laboral experimentado por ellos es mayor en función a los otros grupos de comparación con manifestaciones de desmotivación y descontento interior. 
En otra revisión, Barría (2003) encontró que la extensión de la jornada de trabajo por más de 8 horas en el mismo lugar, incrementa la proclividad al Burnout, como en los casos de los Centros de Diagnóstico, Comunidades Terapéuticas y Centros de Rehabilitación cerrados. Han sido diversas las opiniones sobre el tema, aunque siempre ha centrado el tema de discusión en el estricto sistema de guardias como principal causa del desgaste o Burnout en la realidad española Murillo \& Muñoz, 1991, Jiménez \& Manzano, 1994 (citado pro Zaslove, 2001). En ésta línea, Nichols en el Instituto de Ciencias de la Salud de Oxford (citado por Gil \& Peiró, 1997) encontró que los médicos y abogados son los que presentan mayor incidencia de Burnout, porque es más frecuente en aquellos profesionales que están involucrados en la comunicación con sus pacientes o clientes.

Frente a ello, Wayne (citado por Kenneth \& Davis, 2000), quien ha trabajado con más de 700 médicos que presentan conflicto de trabajo, tanto personal como profesional atribuido al trabajo ético que desempeñan y al fuerte sentido de responsabilidad, les proporciona pautas educativas, ejercicios como caminatas y actividad física, además de conformar grupos de amigos donde se incluyan otras profesiones.

El estudio transversal realizado por Nakata et, al. (2000), a 116 trabajadores japoneses varones de una planta de energía nuclear, los cuales tenian estrés laboral y tensión en el trabajo Se calculó la tasa de concentración de las inmunoglobulinas en la sangre, el número de linfocitos T; observándose en el grupo que tenía más estrés, una baja significativa en ellos en comparación con otro grupo que tenía tensión más baja en el trabajo; se reportó, asimismo, que los estresores psicológicos tal como la muerte de la esposa, divorcio, examen académico, desempleo y cuidado a pacientes con enfermedad de Alzheimer, inducen a la supresión de linfocitos y al incremento de la muerte natural de la actividad celular. También reportan alteraciones en los parámetros inmunológicos en Enfermeras, así mismo se encontró secreción de saliva incrementada en un estudio longitudinal de enfermeras con alto estrés laboral.

A partir de la revisión descrita se planteó el problema sobre cuál es la relación que existe entre el clima laboral, la incidencia del Síndrome de Burnout (Estrés crónico) y el perfil de personalidad predominante asociado a los profesionales asistenciales del área de Hospitalización 13B- 13C, del Dpto. de Neurocirugía del H.N.E.R.M.; el objetivo del estudio es describir las características del clima laboral y su relación con la incidencia de Burnout en los profesionales asistenciales de los servicios de Neurocirugía del HNERM. Así mismo, determinar si existe incidencia de Burnout en los profesionales asistenciales de los servicio de Neurocirugía del HNERM. De igual manera, establecer si existe relación (positiva o negativa) entre los diferentes indicadores específicos del clima laboral: autorrealización, involucramiento laboral, supervisión, comunicación, condiciones laborales en los profesionales y las características de Burnout: agotamiento emocional, despersonalización y falta de realización personal en los profesionales asistenciales del servicio de Neurocirugía del H.N.E.R.M. Finalmente, estudiar el perfil de personalidad que pueda asociarse a este síndrome. 


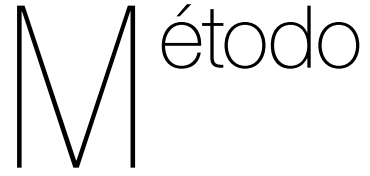

Diseño

El presente trabajo sigue un diseño No experimental, retrospectivo, transversal, y correlacional según la clasificación de Carrasco (2006).

\section{Participantes}

La muestra estuvo conformada por el conjunto de profesionales de Enfermería del Servicio de Columna Vertebral y nervios periféricos. La muestra fue tomada de manera intencional, 30 trabajadores asistenciales, entre 18 enfermeras, todas del sexo femenino y 12 Técnicos de Enfermería, 5 de sexo masculino y 7 de sexo femenino; siendo todos contratados a plazo fijo y tiempo completo, incluido guardias nocturnas. La selección de la muestra fue de tipo no probabilística obteniéndose acceso a ella mediante la disponibilidad de participación y la aceptación del personal, previo a un programa de manejo del estrés que es parte de los talleres de capacitación continua programado para el staff de trabajadores de Neurocirugía; los criterios de inclusión fueron ser profesional asistencial de los servicios de Neurocirugía, aceptación de participación voluntaria, con consentimiento informado, y el tiempo de servicios (1 año y medio mínimo con jornadas ininterrumpidas. En el periodo de evaluación se formó grupos para que tuvieran una evaluación pre-test previo a los talleres y una post-test, luego de haber participado en ellos. Se pretendía conocer si estos talleres mostraban efectividad para el manejo del Síndrome y/o mejoraba el clima laboral. La mayoría de los participantes tenían un periodo de trabajo en el servicio de más de dos años aproximadamente, y la muestra estuvo conformada tanto por personal nombrado como contratado.

\section{Instrumentos}

El Clima Laboral CL-SPN (Palma, 2004), es un instrumento diseñado con la técnica de Likert, de 50 ítems que exploran la variable Clima Laboral definida operacionalmente como la percepción del trabajador con respecto a su ambiente laboral y en función a aspectos vinculados como posibilidades de realización personal, involucramiento con la tarea asignada, supervisión que recibe, acceso a la información relacionada con su trabajo en coordinación con sus demás compañeros y condiciones laborales que facilitan su tarea. Los cinco factores que se determinaron en función al análisis estadístico y cualitativo fueron la autorrealización como la posibilidad que el medio laboral y las funciones realizadas favorezcan el desarrollo personal, con perspectivas a un desarrollo futuro; involucramiento laboral es decir la identificación con los valores organizacionales y compromiso con el cumplimiento y desarrollo de la organización; la supervisión que refiere a la apreciación de la función de supervisión como medio para brindar apoyo y orientación en las tareas que forman parte de su empeño diario; comunicación que es la percepción del grado de fluidez, claridad coherencia y precisión de la comunicación, tanto a 
nivel interno (entre trabajadores), como a nivel externo (clientes y proveedores); finalmente las condiciones laborales entendido como el reconocimiento de que la institución provee los elementos materiales, económicos y/o psicosociales necesarios para el cumplimiento de las tareas encomendadas.

La validez estimada de la escala por el método de jueces y el análisis del poder discriminativo del ítem obtuvo un índice de correlación de .87 y .84 respectivamente. Se estimó la confiabilidad, evidenciando correlaciones de .97 y .90 respectivamente; lo que refiere de una alta consistencia interna de los datos y permite afirmar que el instrumento es confiable.

Inventario de Burnout (MBI) (Maslach \& Jackson, 1997), es una escala cuyos ítems están redactados para expresar sentimientos o actitudes personales y su versión definitiva, pasó por unas fases experimentales que duraron 8 años. Evalúan 3 escalas y 22 ítems distribuidos del siguiente modo: Cansancio emocional (9 ítems), Despersonalización (5 ítems) y Realización Personal (8 ítems). Mientras que en las subescalas de cansancio emocional y despersonalización puntuaciones altas corresponden a altos sentimientos de estar quemado, en la subescala de realización personal en el trabajo bajas puntuaciones corresponden a altos sentimientos de quemarse. Las normas desarrolladas en Perú, en una muestra de profesionales Intensivistas de un Hospital de Lima (Llaja, 2007) se usaron para la presente investigación.

Scanning Neuropsicológico de la Personalidad (Sarria, et.al, 2014), instrumento neuropsicológico evalúa tres áreas principales, las cuales son el Temperamento, Intelecto y Carácter. El temperamento de la personalidad, es organizado por información afectiva consciente (la estructura de sentimientos), del sistema afectivo-emotivo consciente y, que a diferencia del sistema afectivo-emotivo inconsciente que codifica las sensaciones afectivas (sed, dolor), aquel que codifica los sentimientos como son aquellos relacionados con la alegría y la tristeza, el amor y la cólera, la sorpresa y la angustia. En los procesos Afectivo-Emotivos, lo afectivo está constituido por todo un conjunto de disposiciones afectivas, expresan la medida por el cual el hombre, en su interacción con la realidad, logra satisfacer sus múltiples necesidades. Dichas experiencias, básicamente son sentimientos, pasiones y estado de ánimo que se externalizan bajo la forma de gestos emocionales como gritar, llorar, sonreír, abrazar, acariciar, etc.

El Intelecto de modo similar que el temperamento, la estructura psíquica del intelecto está organizada por la información cognitiva consciente (la estructura de conocimientos) que el niño asimila dentro de la estructura cultural de la sociedad, y cuya información nuclear son las aptitudes cognitivas que orientan el desempeño productivo y creativo de la personalidad. Procesos Cognitivo-Productivos; esto procesos se constituyen en nuestras capacidades que nos permiten conocer la realidad.

El Carácter es el componente superior y que más tarda en desarrollarse a lo largo de la historia de la personalidad. Se define como la estructura de la actividad personal que se organiza a partir de la actividad afectivo-ejecutiva de anticipación del infante, del niño y especialmente 
del adolescente, a base de la información conativa que él incorpora dentro de las relaciones sociales económicas del trabajo y la producción, y cuya información nuclear son las actitudes conativas que orientan la conducta volitiva de la personalidad. Este instrumento fundamentado en el Modelo Socio biológico es válido y confiable para realizar la caracterización del perfil asociado a Burnout y al clima laboral en la medida que está adaptado al medio y es el más integral y holístico para describir los perfiles de personalidad encontrados en este papiro.

Así mismo, se realizó un investigación Neuropsicológica (Llaja, et.al., 2014), el objetivo de la investigación fue describir y analizar los trastornos de la actividad psíquica personal de pacientes postoperados de aneurisma cerebral prefrontal; y explicar el compromiso de su actividad funcional mediante el modelo teórico informacional (Ortiz, 1997 p. 62). La muestra estuvo conformada por pacientes diagnosticados por un médico especialista, a los cuales les fue extirpado un aneurisma con la particularidad de ser gigante, y que requirió la ablación de un segmento de esta área y otras técnicas neuroquirúrgicas de esta estructura del neocortex, tan importante para el procesamiento afectivo-emotivo, cognitivo-productivo y conativo-volitivo de la información psíquica consciente del individuo. El muestreo fue no probabilísti$\mathrm{co}$, intencional, y se recogieron los datos durante el periodo de enero del 2009 a mayo del 2010. El diseño fue descriptivo, observacional transversal, y retrospectivo, tipo ensayo clínico según la clasificación de (Díaz, 2009, pp. 34).

Se ha trabajado para conocer en primera instancia la confiabilidad, a través del método de consistencia interna y se ha aplicado el Alfa de Cronbach, el cual fue de .50 lo que indica que tiene confiabilidad alta. Además se calcularon las correlaciones Inter-ítem a través de la medida que la correlación Inter.- ítem promedio determinó la homogeneidad dentro de la escala. Las correlaciones promedio entre los ítems estuvieron entre .15 y .50 .

\section{Procedimiento}

La recolección de la información se hizo en base a Fichas documentales, sobre los participantes de la muestra. Además en cada uno de los protocolos de los instrumentos de evaluación se pudo completar datos académicos, los cuales se tuvieron en cuenta para el tratamiento estadístico.

El análisis de datos se hizo con el Paquete estadístico SPSS versión 20. Se utilizó medidas Paramétricas y No Paramétricas, dentro de lo cual se realizaron los siguientes; se utilizó el estadístico de frecuencias para describir la muestra según instrucción académica, categorías del MBI y Clima laboral.

Posteriormente entrando al propio análisis de datos se buscó conocer la distribución de la muestra que por tener distribución normal se utilizaron estadísticos paramétricos. Para comparar el $\mathrm{MBI}$ y clima laboral se utilizó el estadístico ANOVA para comparar según estado civil cada área del cuestionario de Clima laboral se utilizó el mismo estadístico, y para correlacionar ambas variables se utilizó la $r$ de Pearson. 


\section{Resultados}

En las siguientes Tablas estadísticas se va a detallar cada variable con sus respectivos resultados de frecuencias y porcentajes dándose a conocer en primer lugar los hallazgos en cuanto a proclividad de Burnout, en segundo lugar: Ios niveles del clima laboral encontrado y los hallazgos de correlación de Burnout con los factores de clima laboral. Finalmente los tipos de perfiles predominantes en cuanto a su asociación con Burnout y clima laboral.

Con respecto a la presencia del Síndrome de Burnout en la muestra, encontramos que la mayoría, representando por un $80.0 \%$ no presenta dicho síndrome, mientras que un $10.0 \%$ se encuentra en riesgo, seguido de un 10.0\% que presenta tendencia al Síndrome Burnout (Ver Tabla 1).

Tabla 1.

Descripción de las categorías del MBI

\begin{tabular}{ccc}
\hline & Fr & $\%$ \\
\hline Tendencia MBI & 2 & 10 \\
Riesgo MBI & 2 & 10 \\
No Hay MBI & 26 & 80 \\
Total & 30 & 100.0 \\
\hline
\end{tabular}

De igual manera, con los componentes de dicha variable se encuentra que el $47 \%$ de la muestra presenta indicadores de niveles altos en cansancio emocional, esto puede ser atribuido a que el profesional asistencial de esta especialidad se encuentra constantemente en labores cotidianas que demanda atención oportuna, presión psicológica permanente y decisiones terapéuticas inmediatas por el tipo de paciente y la naturaleza de la enfermedad a la que prestan servicio, igualmente horarios de guardias nocturnas, sobrecarga laboral en otros establecimientos hospitalarios, y otros factores estresantes de la vida familiar y social (Ver Tabla 2).

Tabla 2.

Descripción de las categorías del Clima Laboral

\begin{tabular}{ccc}
\hline & F & $\%$ \\
\hline Desfavorable & 8 & 15.0 \\
Media & 9 & 36.0 \\
Favorable & 11 & 39.0 \\
Muy favorable & 2 & 10.0 \\
Total & 30 & 100.0
\end{tabular}

En cuanto al clima laboral se ha registrado el porcentaje más alto siendo este de 39\% indicando un clima laboral (CL) favorable, seguido de un 60\% indicando un CL con nivel promedio, un $15 \%$ indica un CL desfavorable y un 2\% indica un CL muy favorable (Ver Tabla 3). Teniendo como respuesta a nuestros objetivos que los profesionales asistenciales de salud no se encuentran muy satisfechos con sus responsabilidades laborales, siendo este el $10 \%$, indicando 2 sujetos de la muestra evaluada. Por otro lado, presenta un índice alto de $23 \%$ en el componente de condiciones laborales, significando el reconocimiento por parte de la institución que provee los elementos, materiales, económicos para una mejor labor asistencial. 
Tabla 3.

Descripción de las categorías de satisfacción laboral

\begin{tabular}{cc}
\hline Componentes Satisfacción Laboral & $\%$ \\
\hline Autorrealización & $14.0-$ Media \\
Involucramiento Laboral & $16.0-$ Media \\
Supervisión & $34.0-$ Muy alta \\
Comunicación & $13.0-$ Media \\
Condiciones Laborales & $23.0-$ Alta \\
Total & 100.0 \\
\hline
\end{tabular}

El perfil de personalidad que se asocia con porcentaje respectivamente elevado es: Equilibrado según el Scanning Neuropsicológico (SNP). Esto se debe a que los integrantes de la muestra estudiada son profesionales asistenciales de salud, y los componentes estructurales de su actividad psíquica personal: temperamento, intelecto y carácter se encuentran bajo estándares normativos sin alteración. Este conjunto integrado de doble determinación de los procesos formativos de la personalidad, (determinantes sociocinético y epigenético) corresponden al nivel consciente neocortical de base social. Son individuos transparentes, socialmente equilibrados, empáticos y optimistas. Los rasgos psíquicos de su temperamento los muestran de buena disposición, humor alegre y animado. Las aptitudes cognitivas son individuos eficientes, hábiles, vivaces y competentes, con dominio de habilidades y destrezas ejecutivas para la manipulación o manejo de objetos. Las actitudes motivacionales que orientan la conducta volitiva muestran fuertes valores morales de lealtad y convicción en sus normas éticas y estructura moral de la conciencia (Ver Tabla 4).
Tabla 4.

Perfil de personalidad

\begin{tabular}{cc}
\hline Perfiles clínicos de la Personalidad & $\%$ \\
\hline Personalidad Ansiosa & $13.0-$ Bajo \\
Personalidad Inestable & $14.0-$ Bajo \\
Personalidad Orbito Frontal & $12.0-$ Bajo \\
Personalidad Equilibrada & $48.0-$ Alta \\
Personalidad Orbito Frontal & 13.0 - Bajo \\
Total & 100.0 \\
\hline
\end{tabular}

En la Tabla 5 se puede apreciar que existe una correlación positiva y directa entre el cansancio emocional y Despersonalización, lo que significa que a mayor cansancio emocional mayor despersonalización. Sin embargo el cansancio emocional con autorrealización, involucramiento laboral, supervisión, comunicación, condiciones laborales una correlación negativa e inversa con una $p>05$. Ello indica que a mayor cansancio emocional, menor autorrealización, involucramiento laboral, supervisión, comunicación, condiciones laborales, lo que nos daría características de aquellas personas que presentan un cansancio emocional marcado. Asi mismo existe una relación negativa entre Realización Personal con Cansancio Emocional y Despersonalización.

De igual forma, se observa la correlación negativa entre autorrealización con cansancio emocional con una p>.05, indicando que a mayor autorrealización menor cansancio emocional y correlación positiva con Involucramiento laboral, supervisión, comunicación y condiciones laborales; en lo referente al involucramiento laboral existe relación significativa y negativa $p<.05$ con cansancio emocional y positiva con supervisión, 
comunicación y condiciones laborales; en el área de Supervisión presenta correlación negativa y significativa $\mathrm{p}<.05$ con CE, comunicación y condiciones laborales. Finalmente el área de comunicación presenta una correlación negativa con cansancio emocional $(p<.05)$ y positiva con condiciones laborales y este último así mismo presenta una correlación significativa y negativa con CE. En conclusión, según los hallazgos estadísticos, a mayor intensidad de la Triada Burnout, es proclive a un desfavorable Clima Laboral.

Tabla 5.

Correlación entre las áreas del MBI con el de las áreas evaluadas de clima laboral.

\begin{tabular}{cccccccc}
\hline Áreas del MBI & \multicolumn{2}{c}{ CE } & \multicolumn{3}{c}{ DP } & \multicolumn{3}{c}{ RP } \\
\hline Áreas evaluadas & $\mathbf{R}$ & Sig & $\mathbf{R}$ & Sig. & $\mathbf{R}$ & Sig. \\
\hline CE & 1. &. & $.240\left(^{*}\right)$ & .044 & -.107 & .373 \\
DP & $.240\left(^{*}\right)$ & .044 & 1. &. & -.219 & .066 \\
RP & -.107 & .373 & -.219 & .066 & 1. &. \\
Autorrealización & $-.358\left(^{* *}\right)$ & .002 & .018 & .885 & .083 & .492 \\
Involucramiento laboral & $-.438\left(^{* *}\right)$ & .000 & .014 & .911 & .099 & .413 \\
Supervisión & $-.342\left(^{* *}\right)$ & .004 & -.005 & .969 & .143 & .239 \\
Comunicación & $-.352\left(^{* *}\right)$ & .003 & .081 & .504 & .118 & .332 \\
Condiciones laborales & $-.342\left(^{* *}\right)$ & .004 & .046 & .707 & .193 & .110 \\
\hline
\end{tabular}

En el estudio desarrollado, se ha encontrado un $80.0 \%$ en riesgo, un $10.0 \%$ con tendencia, y la mayoría de la muestra (10.0\% no presenta Burnout). Sin embargo, se debe tomar en cuenta, que riesgo significa, que la muestra estudiada presenta 1 síntoma, y tendencia habla de 2 síntomas presentados; desde este punto de vista preventivo, nos estaría indicando la oportunidad de la evaluación y sugiere la intervención terapéutica con adopción de talleres preventivos de Burnout en el profesional asistencial.

En cuanto al clima laboral se ha encontrado un clima desfavorable, en un $20.0 \%$, que habla de la significación de esta característica en los participantes, que fueron en su mayoría de técnico/ as de enfermería (90.3\%). Así mismo en los hallazgos, no se ha encontrado globalmente una correlación estadísticamente significativa entre los indicadores del clima laboral y el síndrome de Burnout, aun cuando se habla cualitativamente de una correlación inversa entre ambas variables, lo que significa que a mejor clima laboral, menos incidencia de Burnout.

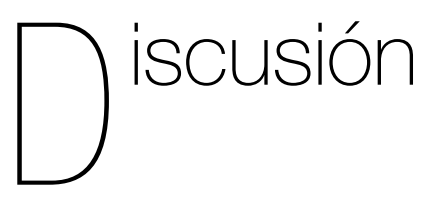

La mayoría de estudios que se han realizado en el medio y a nivel internacional han demostrado la eficiencia de la escala de Maslach \& Jackson para determinar la incidencia de Burnout en muestras de profesionales Asistenciales (Llaja, 
Sarria, \& García, 2007). Así mismo los profesionales de la salud: médicos, enfermeras y tecnólogos médicos, son los que presentan mayor proclividad, como consecuencia de su actividad profesional, para presentar un mayor nivel de estrés o agotamiento profesional (Bulnes, 2004).

Igualmente se habla de correlaciones positivas y negativas entre las variables, llamando la atención que existe correlaciones positivas, estadísticamente significativas, entre las características de Burnout, (por citar correlación positiva entre CE Y DP) y negativas entre RP con CE $Y$ DP que ya han sido demostradas en el estudio anterior citado más arriba .lgualmente se encontró Correlaciones positivas y negativas entre los indicadores de clima laboral desfavorable con características del Síndrome de Burnout (entre ellos: Autorrealización correlación negativa con CE, y positiva con Involucramiento y supervisión. Comunicación, correlación negativa con CE, y positiva con condiciones laborales; así mismo esta última muestra correlación negativa con CE, lo cual confirma el nivel de validez y confiabilidad de estos instrumentos, en cuanto a la determinación de Burnout, y de un clima laboral poco saludable para los profesionales de esta área de trabajo.

Algo esperado, pero no por ello poco significativo, es el nivel de correlación de la variable grado de instrucción, con las áreas de clima laboral. Los hallazgos establecen que presentan mayor involucramiento en sus obligaciones asistenciales, mayor nivel de autorrealización, mejor nivel de comunicación, más eficiencia en la supervisión laboral, en general mejores condiciones laborales, los que tienen grado profesional de enfermería. Al parecer esto podría ser explicado, teniendo en cuenta que la mayor población es profesional de enfermería e incluso realizan horarios de trabajo de guardias diurnas y nocturnas, al no tener estas trabajadoras mayores roles de responsabilidad fuera de la institución donde laboran, determina mejores características laborales en su desempeño profesional, como consecuencia más involucramiento en su rol, y por ende mejores condiciones en el clima laboral y menor incidencia de Burnout.

Estos resultados nos permiten inferir lo vulnerable de la población asistencial estudiada, en ésta y otras investigaciones realizadas, en cuanto que la presión social y psicológica va en aumento, y el estrés crónico se convierte en el flagelo de este siglo, al ser este personal de alto riesgo en Burnout.

Así mismo, si se toma en cuenta en este punto la Teoría de Intercambio social de Blau en (Morales, 1978) se podría afirmar que en el profesional asistencial no médico también juega un rol importante el intercambio social propiamente dicho; vale decir que ellos esperan una relación de reciprocidad y equidad en las relaciones sociales entre personal, paciente e incluso directivos , y que sus actos sean de alguna manera recompensados ; pero al no encontrar ello, hay más probabilidad de que tengan reacciones con sintomatología propia de Burnout. Más aún si observamos que la mayoría de profesionales en este caso de enfermería, adoptan diferentes roles que deben asumir, a parte del rol profesional, en este caso el asistencial, y ello se acentúa aún más al tratarse de personal que trabaja diariamente con pacientes postoperados de cirugía cerebrovascular, y de diferentes patologías 
neuroquirúrgicas, de allí que se hace necesaria la evaluación oportuna y eficaz para estos profesionales, a fin de adoptar medidas preventivas y terapéuticas que alejen la proclividad a Burnout, y la posibilidad de contar a su vez con un clima laboral saludable, donde se observe mayor autorrealización, mejor involucramiento laboral y favorable comunicación interpersonal entre sus miembros.

En lo que se refiere a la explicación de los hallazgos Dek presente estudio bajo el modelo Sociobiológico de la personalidad (Ortiz, 1994) afirmamos una vez más que es el postulado teórico inédito en nuestro medio que interpreta de manera holística e integral la influencia del clima laboral y organizacional en la incidencia del Síndrome de Burnout, vale decir el determinante socio cinético es significativamente prioritario en dicha explicación; así como el epigenético lo es en el perfil de la personalidad de los sujetos examinados.

A partir de estos hallazgos se sugerire hacer mediciones de Burnout y clima laboral periódicas, a fin de establecer programas preventivos de la salud permanentes en los profesionales asistenciales, de estas áreas de trabajo. Planificar y ejecutar estrategias de intervención psicoterapéutica individual o grupal por ciclos (mínimo cada 6 meses) para favorecer un clima antiestrés que beneficie la atención asistencial de nuestros pacientes. Favorecer la cohesión grupal y fortalecer el trabajo en equipo entre los profesionales de Enfermería y Personal técnico a fin de favorecer el clima laboral de la organización y optimizar las actitudes de los responsables del cuidado y recuperación de los pacientes de ésta área asistencial.

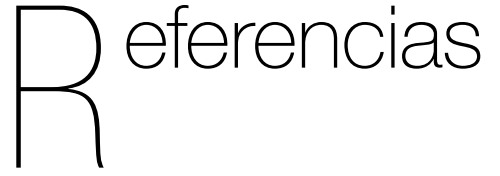

Barria, J. (2203). Síndrome de Burnout en Asistentes Sociales del Servicio Nacional de Menores de la Región Metropolitana de Chile. Tesis de Licenciatura de Psicología. Santiago de Chile: Pontificia Universidad Católica de Chile.

Bulnes, M. (2004). Percepción del clima social laboral y de la eficiencia personal en profesionales de la Salud del Sector Publico de la ciudad de Lima. Revista de Investigación en Psicología, 7(2). 86-89

Capa, L. (2004). Estrés Laboral Asistencial en los Profesionales de la Salud. Tesis de Maestría en Psicología de la Salud. Lima: UNFV.

Carrasco, S. (2006). Metodología de la investigación científica. Lima: Editorial San Marcos.

Civetta, M. (1980). Beyond technology: Intensive care in the $1,980 \mathrm{~s}$. Crit Care Medica, 9, 763-767.

Chernis, C. (1980). El Burnout profesional en las organizaciones de servicios humanos. New York: Praeger.

Dávila, J. \& Romero, P. (2010) Relación entre el Síndrome de Burnout y la percepción sobre el clima organizacional en médicos del área de Emergencia. Tesis de Maestría en Psicología Caracas: Universidad Católica Andrés Bello Recuperado de 2.ucab.edu.ve/anexos/biblioteca/marc/ texto/AAR5044.pdf 
De La Torre, M. (1997). Estrés en Medicina intensiva. Madrid: Sociedad Española de Medicina Intensiva y Unidades Coronarias.

Delgado, A. (2003). El Síndrome del Burnout en Profesores de Educación Secundaria de Lima Metropolitana. Revista de Investigación en Psicología, 6(2), 26- 47.

Dessler, R. (1996). Administración de Personal. Mexico, D.F.: Prentice Hall.

Díaz, M. (2009). Curso Taller avanzado .Técnicas y Métodos de Investigación cientifica . Lima: Manual UPCH.

Villa, L. (2010). Prevalencia del Síndrome de Burnout en alumnos del Séptimo año de Medicina de una Universidad Privada de Lima, Perú. Revista Neuropsiquiatra, 73(4), 147-156.

Fernández, J. (2002). Fuentes de Presión Laboral, Tipos de Personalidad, Desgaste Psíquico (Burnout), Satisfacción Laboral y Desempeño Docente en Profesores de Educación Primaria de Lima Metropolitana. Tesis Doctoral no publicada Facultad de Educación. Lima: U.N.M.S.M.

Fernández, M. (2002). Realidad Psicosocial del Maestro de Primaria. Lima: Universidad de Lima.

Galindo, F. (1993) Relación entre actitudes con respecto a aspectos visibles y aspectos ocultos de la organización de un grupo de trabajadores de un sector industrial de Lima. Tesis de Maestría en Psicología Lima. U.N.M.S.M.

Gamonal, Y., García, C., \& Silva, Z. (2008). Síndrome de burnout en el profesional de enfermería que labora en áreas críticas.
Revista de Enfermería Herediana, 1(1), 33-39.

Gil, P. \& Peiró, J. (1997). Desgaste psíquico en el trabajo: El Síndrome de Quemarse. Madrid: Síntesis.

Gil, P. \& Peiro, J. (1999). Perspectivas Teóricas y Modelos Interpretativos para el Estudio del Síndrome de Quemarse por el Trabajo. Anales de Psicología, 15(2), 261 - 268.

Gil-Monte, P. (2002). Validez Factorial de la adaptación al español del Maslach Burnout Inventory - General Survey. Salud Pública de México, 44(1), 33-40.

Gómez, M. A. (2000). Análisis de contenido cualitativo y cuantitativo: definición, clasificación y metodología. Colombia, Ciencias Humanas, Revista, (20). Recuperado de http://www.utp.edu.co/chumanas/revistas/ revistas/rev20/gomez.htm

Gómez, J. (1991). Los profesionales en la Medicina Intensiva: Aspectos personales, sociales y profesionales del trabajo médico en la UCl. En: Abizanda Campos R. (Ed.) La UCl como centro de responsabilidad, planificación y control, (pp: 191-203.) Madrid: Idepsa.

Guillén, C. \& Guil, R. (2000) Psicología del trabajo para las relaciones laborales. Madrid: Mc. Graw-Hill.

Gutierrez, M \& Saavedra, L. (1999) Clima organizacional, identificación institucional y Expectativas de capacitación en el factor humano de la Dirección de Salud I. Callao: Pinedo Grafic. E.I.R.L.

Hall, R. (1996) Organizaciones, Estructuras, Procesos y Resultados. Mexico, D.F.: Prentice- Hall Hispanoamérica. 
Kenneth, G. \& Davis, M. (2000). Preventing Burnout Family Practice. Management, 7(4), 86- 105.

Llaja, V. \& Muñoz, J. (2000). Abordaje Neuropsicológico en los Servicios de Medicina Intensiva del HNERM. Estudio Piloto. Revista del Cuerpo Médico Hospital Nacional Edgardo Rebagliati Martins, 1(1), 63-87.

Llaja,V. Sarriá , C., \& García, P. (2007). Síndrome del Quemado por Estrés laboral asistencial Muestra peruana. Lima: Pinedo Grafic. E.I.R.L.

Llaja, V., Sarria, J., García, P. (2014). Validación del Scanning neuropsicológico de la personalidad en una muestra clínica de pacientes con trastorno mixto ansiedad-depresión. Revista Psique Mag, 3(1). Recuperado de: http://http:// blog.ucvlima.edu.pe:8080/index.php/ psiquemag/issue/archive

Llaja, V., Sarria, C., Álvarez, L., García, P. (2014). Secuelas neuropsicológicas en pacientes con aneurisma gigante prefrontal postcirugía. Revista Psique Mag, 3(1). Recuperado de: http://http://blog.ucvlima. edu.pe:8080/index.php/psiquemag/issue/ archive

Llaja, V. (2006) Rasgos de Personalidad y el Síndrome de Burnout en el Profesional de Cuidados Intensivos en un hospital de EsSalud de Lima. Tesis Doctoral en Psicología. Lima: U.P.S.M.P.

Maslach, C. \& Jackson, S. (1981). The measurement of experienced Burnout. Journal of Occupational Behavior, 14(2), 99-113.

Maslach, C. \& Jackson, S. (1982). Burnout in health professions: A Social Psychological analysis. En G. Sanders y J. Suls (Eds.) Social Psychology of health and illness, (pp. 56- 68), Hillsdale NJ: Erlbaum.

Maslach, C. \& Jackson, S. (1997). MBI Inventario "Burnout" de Maslach: Manual. Madrid: TEA Ediciones.

Maslach, C. \& Jackson, S. (1986). Maslach Burnout Inventory. Palo Alto, California: Consulting Psychological Press.

Morales, J. (1978). Teoría del Intercambio Social desde la perspectiva de Blau. Revista Española de Investigaciones Psicológicas, 4, 129-146.

Miech, R. (2005). The more interesting and/or consequential your research, the more likely your measures will be contested. Ponencia conferida en el Curso Internacional Métodos de Investigación en Salud Mental y Consumo de Drogas. Lima, Perú.

Nakata, A. (2000). Patterns of Burnout among a national sample of public workers. Journal of Occupational and Enviromment Medicine, 42(2), 176-195.

Orlandini, A. (1996). El Estrés, Qué es y cómo evitarlo. Buenos Aires: Fondo de Cultura Económica de Argentina. S.A.

Ortiz, P. (2004). Introducción a una Psicobiología del Hombre. Perú: UNMSM.

Ortiz, P. (1994). El Sistema de la Personalidad. Lima: Orión.

Ortiz,P. (1997). La formación de la personalidad. Lima: Dimaso Editores.

Palma , S. (2004) Escala Clima Laboral CL-SPC.Manual-1ª. Lima: Editora \& Comercializadora Cartolan E.I.R.L. 
Pezet, J. (1994). Estudio del Clima organizacional en una Empresa en proceso de Cambio. Tesis de Maestría en Psicología Lima: U.N.M.S.M.

Pulido, C. (2003). Clima Organizacional, una Medida del Éxito. Tesis de Maestría en Psicología. Lima: U.N.M.S.M.

Ponce, C., Bulnes, M., \& Aliaga, J. (2001). El Síndrome del "Quemado" por Estrés Laboral Asistencial en grupos de Docentes Universitarios. Memorias del X Congreso Nacional del Psicología. Lima: Colegio de Psicólogos del Perú.

Rubio, E. (Consultora de Empresas). Recuperado de: http://www.gestiopolis. com/canales/derrhh/articulos/42/climaoro. htm

Robbins, S. (1987). Comportamiento Organizacional. México, D.F.: Prentice. Hall.

Rodríguez, D. (1999). Diagnóstico Organizacional. México, D.F.: Alfaomega.

Rodríguez, J. (1995). Psicología Social de la Salud. Madrid: Síntesis Psicologíca.

Rondón P. (1976). Evaluación de grupos de trabajo en relación con la eficiencia de la organización de acuerdo al análisis psicométrico multirrelacional. Buenos Aires: Paidos.

Salvatierra, B. (2000). Orientación Psicológica Laboral. Arequipa: UNSA.

Sánchez, P., \& Sierra, V. (2014). Síndrome de Burnout en el personal de enfermería en UVI. Revista Enfermería Global, 13(1), 252-266.
Sarria, C, Llaja, V., García, P., Quiros, M., Rammuni, G. (2014). Scanning Neuropsicologico clínico de la Personalidad .Lima: Cepredim.UNMSM.

Sarria, C. Llaja, V., García, P. Salazar, M. (2011). Validación del Scanning Neuropsicológico de la personalidad en estudiantes universitarios Lima. Revista de Investigación en Psicología, 14(1), 261-272.

Sarria,C., Llaja V., García,P. (2013). Batería POC-2005. Revisada Sets de evaluación de la actividad Psíquica personal Lima: Cepredim .UNMSM.

Seisdedos, N. (1997). MBI. "Inventario Burnout" de Maslach. Síndrome del "Quemado" por Estrés Laboral Asistencial. Madrid: TEA Ediciones.

Soto, E. (2001). Comportamiento Organizacional. México, D.F.: Thomson.

Solf, A. (1976). Satisfacción Laboral, determinantes y su relación en el desempeño, ausentismo y rotación laboral. México, D.F.: Thomson.

Spielberger, Ch. \& Reheiser, E. (1998). Encuesta de Estrés laboral: Diferencias de Género en la medición del estrés ocupacional. Revista de Psicología II(2) 69-89.

Vásquez, J., Maury, A., \& Verne, E. (2014). Frecuencia del síndrome de Burnout y niveles de sus dimensiones en el personal de salud del servicio de emergencia de pediatría del Hospital Nacional Cayetano Heredia en el año 2014. Lima Perú. Revista de Neuropsiquiatría, 77(3),

Zaslove, M., \& Marshall, M. (2001). Burnout and Emergency Physician Attrition. American Family Physician, 64(3), 553-556. 